\section{Developmental Meuroscience}

Acetylcholinesterase 464

Ag(e)ing 107, 432

Aleuria aurantia lectin 464

Alpha1 subunit 135

Alzheimer's disease 91

$\gamma$-Aminobutyric acid 135

AMPA receptors 122

Androgens 441

Apoptosis 180, 186, 203, 234, 390, 406

ARIA 25

Asphyxia 175

Astrocyte(s) 299, 399

Axonal signal 25

- transport 364

Basal ganglia 91

Bax 186

Bicistronic vector 145

Brain 171

- damage 171, 192

- development 198, 219,412, 452

- injury 213

- slice 412

Branchial motoneurons 113

Bromodeoxyuridine 346

\section{cAMP 70}

Cardiac myocytes 153

Cardiovascular responses 175

Caspase(s) 186, 203

Catecholamine 432

Cell cycle 100

- death 203, 234, 406

- lines 153

Cerebellar granule cells 145

Cerebellum 31

Cerebral atrophy 180

- blood flow 209

- glucose 219

- palsy 203, 213, 234

Cerebrovascular circulation 224

- responses 175

Chemokine 338

Chick embryo 346

8-(4-Chlorophenylthio)-3',5'-cyclic monophosphate 224

Choline 100

Chromatography 107

CNS hypomyelination 7

Conditionally immortalized cell lines 452

Congenital lesion 171

Connexins $(36,43) 420$
CXCR2 338

2'-3'-Cyclic nucleotide 3'-phosphodiesterase 287

Cyclins 356

Cyclophilin 135

Cytochrome $c$ oxidase 186

Cytokeratin K19 452

Dentate gyrus 100

Development 1, 91, 338, 377, 390

Diazepam 135

Differentiation 377

- factor (neu) 25

DNA damage 432

Dopamine 78

Double mutant mice 7

Electroporation 346

Embryonic brain 318

- development 70

Enhanced green fluorescence protein 346

Enolase 406

Epidermal growth factor receptor 84

Epilepsy 219

Epithelium, pigmented 268

erbB 377

erbB2 25

erbB3 25

erbB4 25

Excitatory amino acids 412

Fas death receptor 186

Fatty acids $\left(\mathrm{C}_{18}\right) \quad 107$

Fetal sheep 175

Folch extraction 107

Free radical 412

Gap junctions 420

Germinal matrix 234

Glia 25

Glial cell differentiation 452

- fibrillary acidic protein 299

- growth factor 25,377

- precursor cells 277

- proteins 84

Gliogenesis 287

Glucose metabolism 219, 412

Glutamate 41

- receptors 31

- toxicity 399

Glutathione 198

- peroxidase 198
Glycolipid antigens 346

Glycoproteins 464

Green fluorescent protein 287, 346

GRO1 338

Guanosine 3',5'-cyclic monophosphate 224

Guanylate cyclase 224

Heregulin 25, 377

Hippocampus 31,100

Homeobox gene 17

$5-\mathrm{HT}_{1 \mathrm{~A}} 70$

$5-\mathrm{HT}_{4} 70$

Human fetal hippocampus 420

- neuroectodermal cells 84

Hydrogen peroxide 198

6-Hydroxydopamine 78

Hypothyroidism 277

Hypoxia 171, 180, 198, 406, 412

Hypoxia-ischemia 192

Immature rats 192

Immunocytochemistry 91

Immunohistochemistry 41,78

Immunoreactivity 406

Immunostaining 441

Infection 213

Insulin-like growth factor II 70

- _ factors 70

Iron 198

- deficiency 277

Ischemia 171, 180, 198, 209

Isobutylmethylxanthine 70

Jaw 41

Kainate receptors 122

Kinesin regulation 364

Lentiviral vector 145

Leukocyte inhibitory factor 327

Mandible 70

Maturation, neurons 406

Memory 100

Metabotropic glutamate receptor 1 mitf 268

Mitochondria 186, 192

Mitogen 25

mRNA 135

Myelination 7, 25, 287, 327, 377
KARGER

(C) 2002 S. Karger AG, Basel

Fax + 41613061234

E-Mail karger@karger.ch

www.karger.com
Accessible online at:

www.karger.com/journals/dne 
Necrosis 406

Neostriatum 78

Nerve growth factor 55

Neuregulin 25, 327, 377

Neurite extension 55

- outgrowth 364

Neurodegeneration 432

Neuroglia 203

Neuromuscular junctions 441

Neuronal development 364

Neurons 406

Neuron-to-astrocyte signaling 420

Neuroprotection 31

Neurotrophic factor 145

Neurotrophin 390

NGFI-B family 17

NMDA receptors 122

Non-A $\beta$ component, amyloid 91

NOR-1 17

Nutrition 100

Oligodendrocyte(s) 203, 234, 277, 377

- development 327

- lineage 452

- precursors 338

- progenitors 287,318

- proliferation 356

Oligodendroglia 452

Orphan nuclear receptor 17

p13 $3^{\text {sucl }} 55$

p27Kip1 356

p75 390

Parkinson's disease 91, 432 pax6 268

Pax-6 464

PC12D cells 55

Perchloric acid extraction 107

Perinatal asphyxia 213

- brain 171

- - damage, pathophysiology 171

- hypoxia-ischemia 180

Permeability transition 192

Phosphorylation 364

Photoreceptors 464

Pigment epithelium derived factor 145

Platelet-derived growth factor 327

Positional factors 318

Pregnancy 100

Prematurity 175

Preterm infant 209

Programmed cell death 432

Protein kinase $\mathrm{C} 55$

- _ inhibitor 70

- tyrosine phosphatase $\zeta 55$

Proteolipid protein(s) 287, 299

Reaggregation 464

Receptor-like protein tyrosine phosphatase $\gamma 55$

Regeneration 268, 377

Remyelination 377

Retina 268, 390, 464

Retinospheroids 464

Rp-cAMPS 70

Rp 8-(4-chlorophenylthio)-3',5'-cyclic monophosphorothioate 224

Rumpshaker 7
Serotonin 78

Sexual dimorphism 399, 441

Shiverer 7

Six 317

SPECT 209

Spectroscopy 213

Striatum 1,122

Stroke 203, 234

Subtraction library 452

Suckling 41, 113

Sympathetic neurons 153

Synapse stabilization 441

Synaptogenesis 153

$\mathrm{T}_{3} 327$

Tat 145

T-cadherin 452

Temporal lobe 219

Thyroid hormone 277

Transdifferentiation 268

Trigeminal motor nucleus 113

Type A $\gamma$-aminobutyric acid receptor 135

Tyrosine hydroxylase 406

Vimentin 299

White matter 209

Xenopus laevis 299 